\title{
Proceeding
}

\section{Effectiveness of motivational videos for elite swimmers: Subjective and biological evaluations}

\author{
GOICHI HAGIWARA ${ }^{1}$, DAISUKE AKIYAMA², TAKAAKI TSUNOKAWA ${ }^{1}$, HIROTOSHI MANKYU1 \\ ${ }^{1}$ Faculty of Physical Education, Department of Sports Humanities and Applied Social Science, National \\ Institute of Fitness and Sports in Kanoya, Japan \\ ${ }^{2}$ Faculty of Human Science, Department of Sport Science and Health, Kyushu Sangyo University, Japan
}

\begin{abstract}
Motivational videos (MVs) are used in Japan in many competitive sports, both professional and amateur. However, several researchers have stated that the effectiveness of MVs has not been scientifically verified, and only subjective evaluations have been conducted in previous studies (Yamazaki \& Sugiyama, 2009). This study examined the psychological effectiveness of MVs using both subjective and biological evaluations. The subjects were six elite Japanese collegiate swimmers participating in the FINA Swimming World Cup (age: $21.33 \pm 0.51$, four males and two females). We employed subjective and biological evaluations to examine these elite swimmers' psychological motivation while watching an MV as part of their practice. We adopted subjective and self-report Psychological Condition Inventory (Yamazaki et al., 2008) to measure the athletes' level of vigor while watching the MV. An electroencephalogram (EEG) was employed to evaluate the emotions of the swimmers. The emotional condition (vigor) was analysed by combining the extracted raw EEG data using a KANSEI module logger (Littlesoftware, 2018). A subjective evaluation showed that the score for vigor significantly improved after watching the MV $(t=2.33, p<0.05)$, which was also indicated by the results of the biological evaluation $(t=5.69, p<0.01)$. There was also a significant correlation between subjective and biological vigor after watching the MV $(r=0.77, p<0.01)$. The results of this study, therefore, indicated that biological evaluation can be employed anew to verify the effectiveness of MVs. Key words: EEG; Motivation in sports; Performance improvement; KANSEl.
\end{abstract}

Cite this article as:

Hagiwara, G., Akiyama, D., Tsunokawa, T., \& Mankyu, H. (2019). Effectiveness of motivational videos for elite swimmers: Subjective and biological evaluations. Journal of Human Sport and Exercise, 14(2proc), S178-S188. doi:https://doi.org/10.14198/jhse.2019.14.Proc2.04

Corresponding author. Faculty of Physical Education, Department of Sports Humanities and Applied Social Science, National Institute of Fitness and Sports in Kanoya, 891-2393, Shiromizu-cho, Kanoya-shi, Kagoshima, Japan.

E-mail: hagiwara-g@nifs-k.ac.jp

Supplementary Issue: Winter Conferences of Sports Science. Costa Blanca Sports Science Events, 25-26 January 2019. Alicante, Spain.

JOURNAL OF HUMAN SPORT \& EXERCISE ISSN 1988-5202

(c) Faculty of Education. University of Alicante.

doi:10.14198/jhse.2019.14.Proc2.04

S178 | 2019| Proc2 | VOLUME 14

(C) 2019 University of Alicante 


\section{INTRODUCTION}

A motivational video (MV) is defined as a video that is produced by extracting only the best performance from the recordings of past games of the target athletes and teams, and adding music and text to it (Nagao, 2010). Its aim is to alter a player's psychological outlook, deemed necessary for him/her to demonstrate good performance in upcoming games, matches or training, to a positive state (Halliwell, 1990; Nagao \& Sugiyama, 2013; Yamazaki \& Sugiyama, 2009). Yamazaki and Sugiyama (2009) conducted an experiment with ten university badminton players to verify the effects of an MV. They showed that watching a video yielded psychological benefits, such as gaining subjective fighting spirit and becoming vigorous. These subjects showed a significantly high success rate of shots than did the group of subjects who did not watch the MV. Hence, watching MVs has been known to affect both psychological outlook and performance, and is increasingly being adopted in competitive sports, in Japan in both professional and college sports.

Halliwell (1990) conducted a study that targeted NHL players. He produced a video compilation of clips of their best performance during a game and added their favourite music to the compilation. He then investigated the relationship between viewing the video and the subjects' subsequent performance. The results showed that watching the video enhanced the performances of the target players, and that it led to an increase in their confidence. Numerous scientific verification experiments have used such methods; however, researchers have pointed out that these practical activities and studies lack theoretical or scientific evidence of their efficacy, and that the type of effects they produce are not very clear (Yamazaki \& Sugiyama, 2009). Although subjective evaluations have been carried out using questionnaires given to athletes before and after watching an MV (Yamazaki \& Sugiyama, 2009, Nagao \& Sugiyama, 2013), no objective evaluations of the psychological effects of such MVs have yet been attempted.

Subjective evaluations are consistently alleged to suffer from individual biases, which result from the differences in the way the words of individual subjects are interpreted (Takano et al., 1993). An evaluation method using biological data, on the other hand, is likely to be more objective, since the measurement values are less likely to fluctuate because of human bias (Nagamatsu \& Nakazawa, 1999). Another advantage is that, since measurements can be made in real time, changes in the time series can be identified (Nagamatsu \& Nakazawa, 1999). Biological evaluation can therefore be said to be more effective in verifying the psychological effects of MVs.

The electrical activity in the brain is often used as an objective evaluation index that employs biological data. Brainwaves are generally classified into four types according to their frequency range $(0.5-4 \mathrm{~Hz}$ : delta waves; 4-8 Hz: theta waves; 8-13 Hz: alpha waves; 13-40 Hz: beta waves), and emotions can be associated with each type (Okubo, 2018) (Table. 1). During human activity, a frequency exceeding $4 \mathrm{~Hz}$ appears. To measure emotions during human activity, therefore, alpha and beta waves are used. Hence, human emotions are measured using frequency wave bands. It has been suggested, however, that judging emotions using only frequency wave bands, as described above, is far too speculative, and that the method cannot reveal a person's emotions adequately (Mitsukura, 2016). Methods are therefore being proposed for assuming human emotions by segmenting the frequency wave bands (Shibata et al., 2015), and using a combination of the appearance ratios of a particular frequency wave band (Okubo, 2018; Mitsukura, 2016).

Mitsukura (2016) defined emotions such as stress, concentration, drowsiness, interest, and likes/dislikes using a combination of frequencies appearing in EEGs, and showed that it can be utilized as an index for evaluating emotions. Yoshida et al. (2012) also noted that the degree of vigor and activity of the human brain can be measured using a combination of alpha and beta waves. Researchers have also investigated the use 
of a combination of frequencies to infer the degree of satisfaction (Matsunaga \& Nakazawa, 1999); emotions such as delight, anger, sorrow, and pleasure (Musha et al., 1997); and favour/goodwill (Fukai et al., 2013). It is now possible to express human emotions in terms of a combination of brain frequencies.

Table 1. Type of brain wave

\begin{tabular}{|c|c|c|}
\hline Type of brain wave & Frequency $(\mathrm{Hz})$ & Psychological status \\
\hline Delta wave & $0.5 \sim 4 \mathrm{~Hz}$ & non-REM sleep, unconscious \\
\hline Theta wave & $4 \sim 8 \mathrm{~Hz}$ & Sleep onset, illusion \\
\hline Alpha wave & $8 \sim 13 \mathrm{~Hz}$ & Relax \\
\hline Beta wave & $13 \sim 40 \mathrm{~Hz}$ & Arousal \\
\hline
\end{tabular}

The purposes of this study were to investigate the psychological effects of MVs using both subjective and biological evaluations, verify the psychological changes that occur with time in the viewer while watching an $\mathrm{MV}$, and identify the relationship between watching an MV and subsequent performances.

\section{METHODS}

\section{Subjects}

The subjects were six elite Japanese collegiate swimmers participating in the FINA Swimming World Cup (age: $21.33 \pm 0.51$, four males and two females). The research team informed the subjects of the purpose of the study and gave instructions on the experiment prior to participation. Data and informed consent were obtained from the participants of this study. Participation was entirely voluntary.

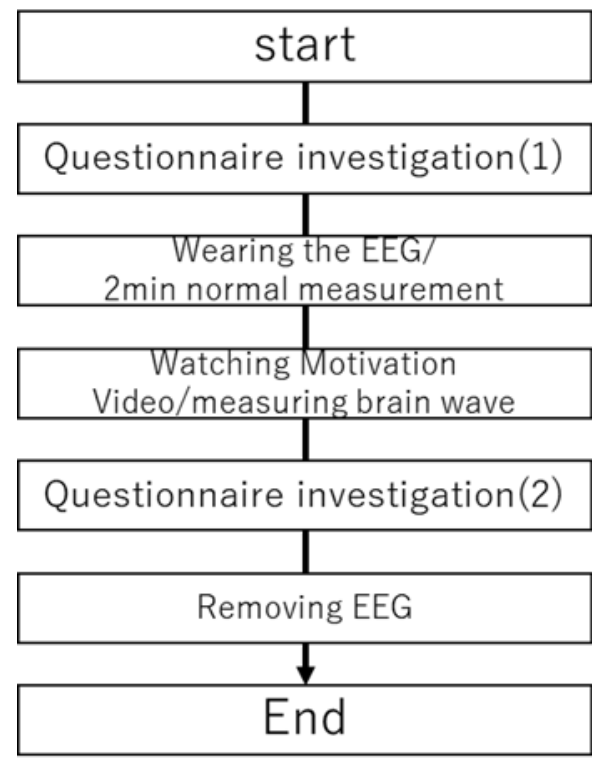

Figure1. Procedure

\section{Procedures}

The subjects were instructed to gather in a meeting room (MR) close to the pool, 90 minutes before the start of a time trial. After being informed of the outline and risks of the study, and obtaining their consent, they 
were asked to sit down and answer a questionnaire (No. 1). Subsequently, they were asked to put on an EEG device and undergo two minutes of calibration to measure their baseline degree of vigor during normal periods. Later, they watched an MV and answered the second questionnaire (Figure 1). The subjects then participated in a time trial.

\section{Content of an MV}

An MV was edited by extracting clips featuring scenes from the subject players' practice and races, and suitable music was selected. Editing was done with a video editing application that works on Macintosh (iMovie ver. 2.2.5). Since a previous study (Yamazaki et al., 2008) has shown that videos should ideally be viewed 90 minutes before the start of a match, we made the subjects watch the video 90 minutes before the start of their time trial. Regarding the length of the video, Dowrick (1991) reported that 2-5 minutes' content minimizes the time burden on the subjects while having a maximum effect. For this study, we therefore created an MV lasting 4 minutes and 19 seconds.

\section{Evaluating psychological motivation}

Subjective evaluations

To subjectively evaluate the subjects' psychological outlook, we used "general vigor," which is a motivationrelated factor that features in the Psychological Condition Inventory for MVs (PCl) drawn up by Yamazaki et al. (2008). The reliability and appropriateness of this scale have been confirmed by Yamazaki et al. (2008). The range of scores that can be obtained on this scale is 4 to 20 points. Higher scores indicate a better psychological condition on the part of the subject. In a previous study that used this scale (Yamazaki et al., 2008), data were collected before and after watching an MV, with the scores for general vigor reported to have risen after watching it. The results also suggested a relationship between watching such videos and subsequent performance.

\section{Biological evaluations}

This study conducted biological evaluations of the effects of MVs in the form of an EEG to evaluate the emotions of elite swimmers. We adopted a simple band-type EEG (Fig. 2) that only measures the Fp1 point as defined by the international 10-20 system (Fig. 3). Since Fp1 is located on the left frontal lobe, there is no need to worry about the possible noise interference caused by hair; and the EEG obtained from Fp1 has been shown to be suitable for obtaining data on people's psychological condition (Mitsukura, 2016; Hotta et al., 2017). We obtained data on the intensity of alpha and beta waves at one-second intervals by having the subjects wear an EEG recorder on their heads, and attaching the electrodes to the ears and forehead. Intensity was calculated by subjecting the EEG's raw data to Fourier transform. The data thus obtained were recorded in a smartphone, and a Kansei Module Logger (Littlesoftware Inc., 2018) that can output the data as sensitivity values was used. This study used vigor as an index since it shows an emotional pattern similar to "general vigor," which is used in subjective evaluations. We measured the intensity of vigor according to the magnitude of the subjects' "general vigor." In converting the sensitivity values, we used the obtained data as the basis for cutting out wave shapes from the frequency wave bands ranging from $0.5 \mathrm{~Hz}$ to $40 \mathrm{~Hz}$, and analysed them, using an algorithm. Here, regarding vigor, we used data that had been extracted from alpha waves $(8-13 \mathrm{~Hz})$ and beta waves $(13-40 \mathrm{~Hz})$, which are brain wave frequency wave bands that become prominent during human activity. We define vigor by calculating the occurrence ratio, especially from beta waves, which are produced when a person is in an alert state. Vigor is shown in numbers ranging from 0 100 , and the higher the numerical value, the higher is the degree of vigor. 


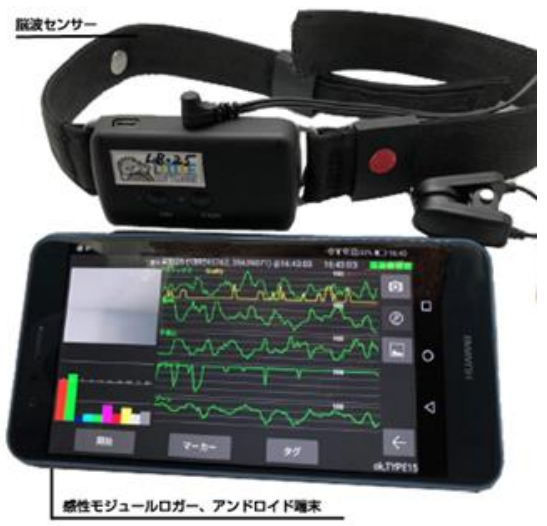

Figure 2. Band-type EEG and KANEI module logger

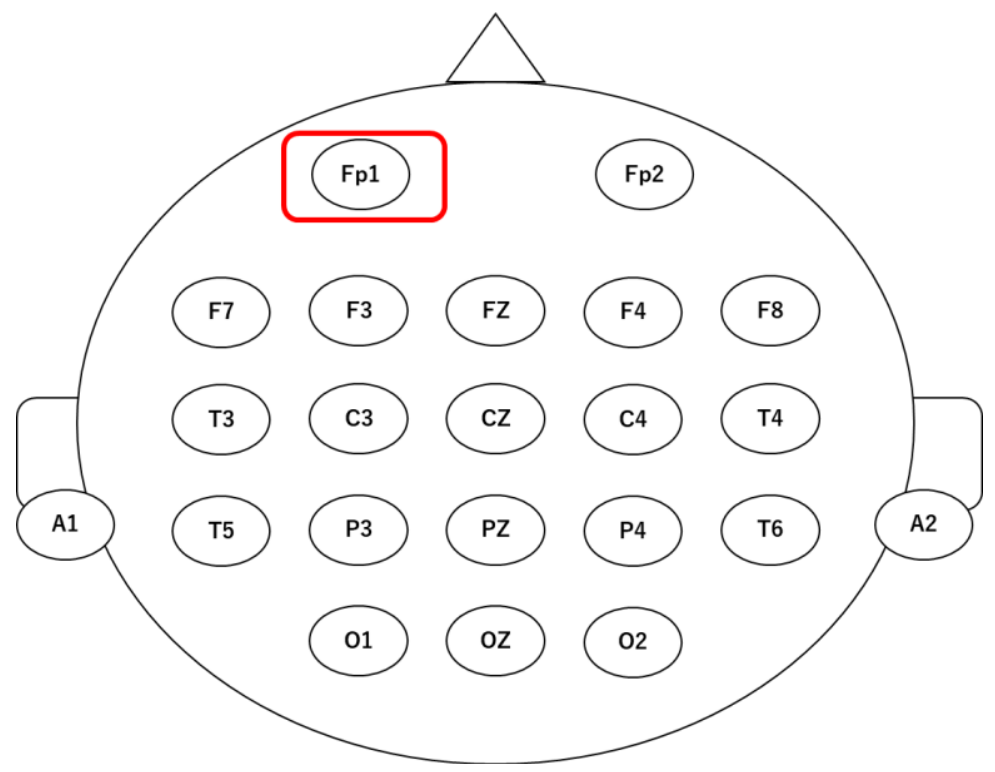

Figure 3. Measurement points of the international $10-20$ system

Measures of performance

As measures of performance, we used the best ratio of the time recorded at a time trial. The best ratio (\%) was calculated using the personal best time of a subject, and his/her time recorded at a time trial. It is calculated by dividing the time recorded at a time trial by the subject's personal best time, multiplied by 100 . The closer a figure is to $100 \%$, the higher the best ratio is evaluated, and the higher the numerical value gets than $100 \%$, the lower the best ratio is evaluated. Table 2 shows the best ratios of the subjects' time trial conducted in this study.

\section{Analysis}

For objective data, the results of a questionnaire survey conducted before and after watching MVs were compared, using a paired $t$-test. For biological data, we used the paired $t$-test to compare the average values of vigor measured during normal periods and the average value of vigor measured when watching the MV. Moreover, to verify the correlation between objective and biological data, we calculated the correlation coefficient of the average value of the results of the questionnaire survey conducted after watching the video, 
and the average value of EEG data measured while the subject was watching the MV. We then designated subjects having the best ratios, calculated via performance measurements of around $100 \%$, as the Higher Ratio Group, and those having the best ratios exceeding 102\%, as the Lower Ratio Group, and performed a paired $t$-test, using the degree of biological vigor after watching the MVs as the dependent variable. IBM's SPSS24.0 statistical software was used for the analysis. With time-series data, meanwhile, we used Microsoft Excel 2016 to draw up a graph of the degree of vigor that is being calculated.

Table 2. Best time rates for swim trial

\begin{tabular}{|c|c|c|c|c|c|}
\hline Subject & Gender & Swim event & Best time & Time trial & Best time rates \\
\hline A & Male & Freestyle $(200 \mathrm{~m})$ & 1'52"'29 & 1'55"32 & 102.6 \\
\hline B & Male & Backstroke $(100 \mathrm{~m})$ & $57 ” 00$ & $57 " 46$ & 100.8 \\
\hline $\mathrm{C}$ & Male & Individual Medley(200m) & 2’05”40 & 2’08”05 & 102.1 \\
\hline $\mathrm{D}$ & Male & Butterfly stroke(200m) & 1'59”61 & 2’00”70 & 100.9 \\
\hline $\mathrm{E}$ & Female & Freestyle(200m) & 2’04”21 & 2’04”31 & 100.0 \\
\hline $\mathrm{F}$ & Female & Butterfly stroke(100m) & $59 ” 51$ & 1’02”32 & 104.7 \\
\hline
\end{tabular}

\section{RESULTS}

\section{Comparison of subjective data collected before and after watching an MV}

We analysed the scores on various scales before and after watching an MV using a paired $t$-test, and found a significant difference in "general vigor" before watching an MV $(M=13.16, S D=2.48$ ) and afterwards ( $M$ $=15.16, \mathrm{SD}=1.16)(t=2.33, p<0.05)$ (Figure 4). Enhanced subjective vigor was noted after watching an MV compared to before watching it.

\section{Subjective Vigor}

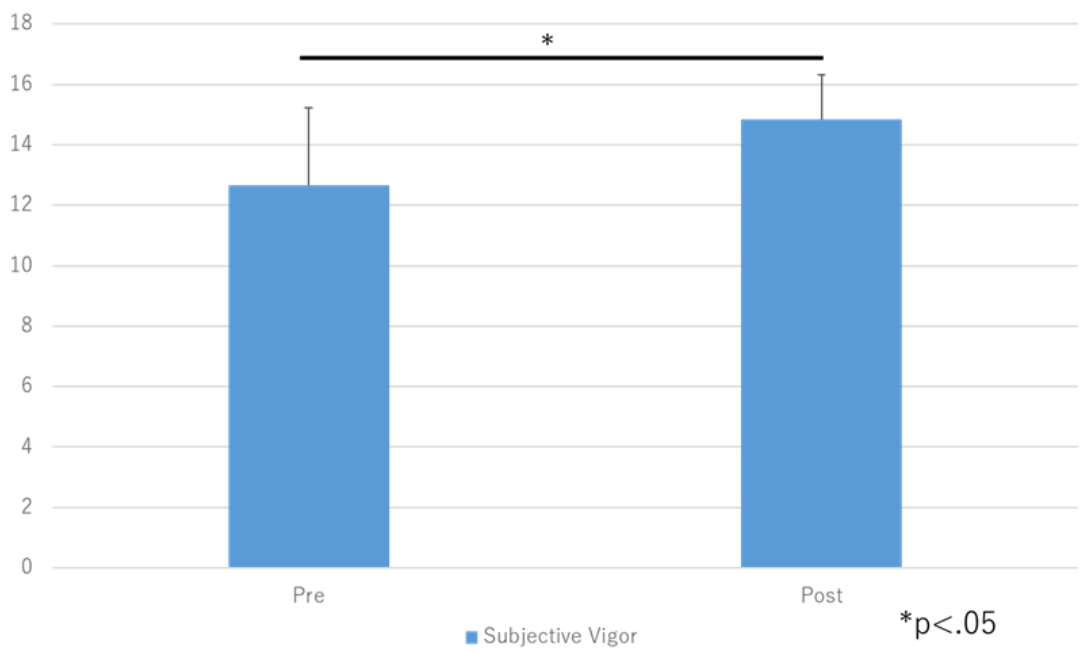

Figure 4. Results of t-test for subjective vigor

\section{Comparison of biological data collected before and after watching a video}

A paired $t$-test was performed on the average value of the degree of vigor in normal periods and while watching an MV. The results indicated a significant difference between normal periods $(M=31.26, S D=$ 
3.15) and while watching an MV $(M=37.50, S D=4.58)(t=5.69, p<0.01)$ (Figure 5). Higher biological vigor was noted while watching an MV compared to the period before watching it.

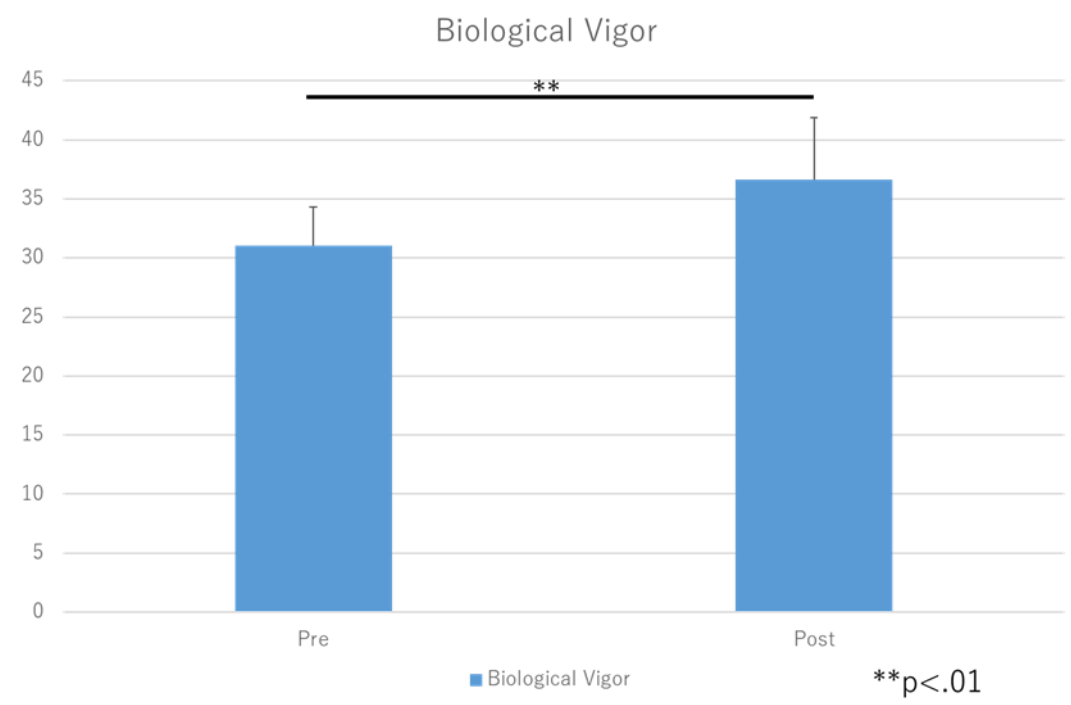

Figure 5. Results of t-test for biological vigor

\section{Correlation between subjective and biological data}

We calculated the correlation coefficient between the average value of the results of a questionnaire that was answered after watching a video, and the average value of EEG data calculated while watching an MV, and found a significant positive correlation between the two (Table 3).

Table 3. Correlation between subjective and biological vigor

\begin{tabular}{|c|c|c|}
\hline & Subjective Vigor & Biological Vigor \\
\hline Subjective & - & $.768^{*}$ \\
\hline Biological & $.768^{*}$ & - \\
\hline
\end{tabular}

Time-series data of biological activity while watching an MV

Figure 6 shows the time-series data of the subjects' biological vigor while watching an MV. It was revealed that the timing at which the degree of vigor increased differed depending on the subject. 


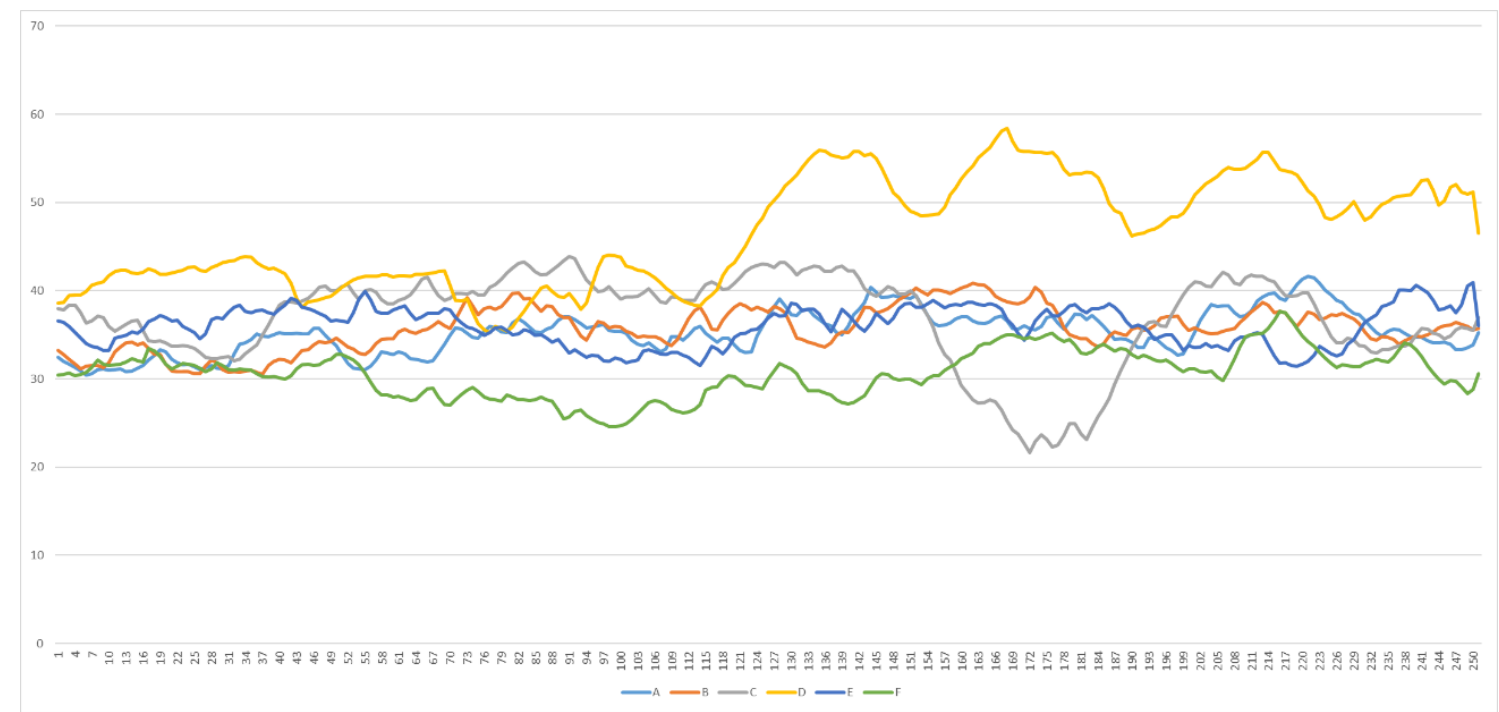

Figure 6. Time-series data in chronological order

\section{Relationship between performance and biological vigor}

We designated the subjects having the best ratios, calculated via performance measures, of around $100 \%$ as the Higher Ratio Group, and those having the best ratios exceeding $102 \%$ as the Lower Ratio Group. We then conducted a paired $t$-test, using the degree of biological vigor after watching the MV as the dependent variable. The results showed a significant tendency among the two groups $(t=1.96, p<0.10)$. It was found that those in the higher ratio group ( $M=42.56, S D=7.95$ ) tended to have higher biological vigor after watching an $\mathrm{MV}$ than those in the lower ratio group $(\mathrm{M}=32.72, \mathrm{SD}=3.47$ ) (Figure 7 ).

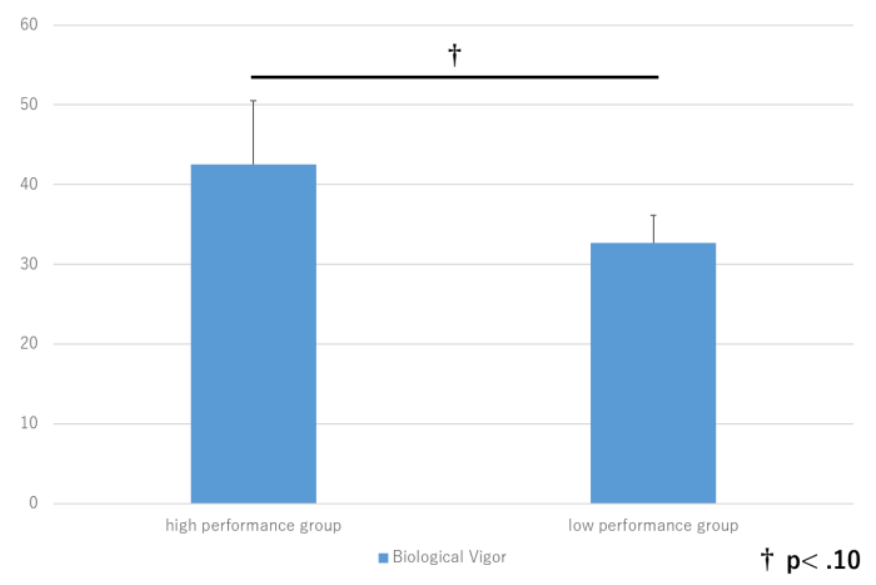

Figure 7. Results of t-test for biological vigor between higher and lower ratio groups

\section{DISCUSSION}

This study aimed to investigate the psychological effects of an MV using both subjective and biological evaluations, verify psychological changes in the subjects while watching an MV, and identify any relationship between watching the MV and the subjects' subsequent performance. 
Regarding subjective symptoms, the scores on the scales before and after watching the MV were compared. Regarding "general vigor," the results showed that "subjective vigor" significantly increased after watching MVs. These results lead us to conclude that we were able to obtain the results similar to those of previous research (Yamazaki et al., 2008; Yamazaki \& Sugiyama, 2009). Next, regarding biological evaluations, we used EEG to compare the average values of vigor during normal periods and while watching an MV, and found that the degree of vigor was significantly higher while watching an MV compared to that during normal period (before watching an MV). In other words, this study can be said to have revealed biologically the psychological effects of viewing an MV. Previous research (Yoshida et al., 2012) similarly revealed that the degree of vigor and activity of the human brain can be measured by using a combination of alpha and beta waves. Since this study has provided new knowledge in the field of sports, it is hoped that, going forward, athletes' vigor can be measured using EEG.

A correlation coefficient was calculated between the average value of the results of a questionnaire survey conducted after watching a video, and the average value of EEG data that were calculated while watching MV. We found a significant positive correlation between the two. In other words, the method used in this study is proven to be valid. Previous studies (Fukai et al., 2013; Mitsukura, 2016) have also investigated the relationship between subjective and biological evaluations, and attempted to show the validity of emotional evaluations using EEG. Since a positive correlation was shown in this study, we believe that our results will contribute to the further development of research pertaining to emotional evaluations using EEG. However, we cannot state unequivocally that we are able to confirm the validity of EEG-based evaluations solely from the results of our study. We, therefore, believe that it is desirable to continue conducting investigations by, for example, increasing the number of samples.

The time-series data on the degree of biological vigor while watching an MV showed that the timing at which vigor increased differed according to the subject. For example, in subject $D$, vigor increased at 115 seconds when scenes with his/her teammates' messages and cheering began to be showed, and a high level of vigor was maintained until the end of the video. In subject $C$, on the other hand, vigor started declining at 135 seconds, and conversely, rise again at 180 seconds when scenes of his/her teammates and himself/herself swimming at meets were shown. These observations suggest that the content of the MV shown to individual subjects had influenced his/her degree of vigor. Nagamatsu and Nakazawa (1999) stated that the evaluation method that employs EEG is objective since the measurement values are less likely to be subject to varying human interpretations, and that it is possible to grasp variations with the elapse of time. Therefore, it can be said that our method is effective in investigating the psychological effects of MVs in the field of sports.

We compared biologically-determined vigor after watching the MV in the subjects in the Higher Ratio Group and the Lower Ratio Group, which were formed on the basis of performance measures, and found a significant difference between the two groups: the group with the high best ratios tended to have higher biologically-determined vigor after watching MVs than the group with low best ratios. Yamazaki and Sugiyama (2009) investigated, using university badminton players as their subjects, the relationship between subjective vigor while watching MV, and the ratio of executing successful shots in badminton. The results showed that the greater increase in subjective vigor in a player while watching an MV, the better his/her subsequent performance. The results of the present study, which used biological data, showed a relationship between increase in subjective vigor while watching an MV and the subsequent performance. Therefore, it will most likely be possible to investigate the effects of watching MVs in the future, using EEG. On the other hand, there were limitations as to the data samples since our study investigated only top-level athletes. We measure that by conducting investigations involving players at different levels, such as university athletes, it will be possible to obtain data that can be further generalized. 


\section{CONCLUSION}

In conclusion, the results of this study, which used both subjective and biological evaluations, support the psychological effectiveness of MVs. The results of subjective evaluation showed significant improvement in the score for vigor after watching the MV. The results of biological evaluations also indicated significantly improved vigor after watching the MV. There was a significant correlation between subjective and biological vigor after watching MVs, and this study showed a relationship between performance and biological vigor. The results of this study, therefore, indicated that biological evaluation can be employed anew to verify the effectiveness of MVs.

\section{REFERENCES}

Dowrick, P. W. (1991). Practical guide to using video in the behavioral science. New York, J. Wiley.

Fukai, H. Tomita, Y. \& Mitsukura, Y. (2013). A design of the preference acquisition detection system using the EEG. Int. J. Intelligent Information Systems, Vol. 2, No. 1, 2013, pp. 19-25. https://doi.org/10.11648/i.ijiis.20130201.13

Halliwell, W. (1990). Providing sport psychology consultant services in professional hockey. Sport Psychol., 4, 369-377. https://doi.org/10.1123/tsp.4.4.369

Hotta, M. \& Kohata, Y. (2017). The evaluation of usability of EC site using electroencephalogram (EEG). Proc. 19th Jpn. Soc. Kansei Eng. 1-5.

Littlesoftware (2018, November 10). Kansei Module logger. Retrieved from http://www.littlesoftware.jp/product/

Matsunaga, H. \& Nakazawa, H. (1999). An attempt to measure human satisfaction by EEG measurement. Trans. Jpn. Soc. Mech. Eng. Ser. C. 65, 2135-2140. https://doi.org/10.1299/kikaic.65.2135

Mitsukura, Y. (2016). KANSEI Analyzing by EEG. J. Inst. Electr. Eng. Jpn. 136, 687-690. https://doi.org/10.1541/ieejijournal.136.687

Musha, T., Terasaki, Y., Haque, H. \& Ivamitsky, G. (1997). Feature extraction from EEGs associated with emotions. Artif. Life Robot. 1, 15-19. https://doi.org/10.1007/BF02471106

Nagao, Y. \& Sugiyama, Y. (2013). Influence on a collective efficacy of viewing a motivational video of a game in relation to type of video. Res. J. Sport Perform. 5, 352-368.

Nagao, Y., Sugiyama, Y., Yamazaki, M., \& Kawazu, K. (2010). The resources and application of collective efficacy in team sport. J. Health Sci. 32:11-19. https://doi.org/10.15017/18336

Ohkubo, T., Tamamaru, K. \& Koshimizu, S. (2018). Development of the Impression Detection System by using a Portable EEG Device for Tourist Impression Analysis. Trans. Jpn. Soc. Kansei Eng. 17, 285291. https://doi.org/10.5057/jijske.TJSKE-D-17-00082

Shibata, S., Akita, T. \& Kimura, H. (2015). Emotion extraction method for listening to the pleasant and unpleasant sound using alpha and beta wave on a simplified EEG. Bull. Daido Univ. 51, 97-103.

Takano, K., Nagasaka, A. \& Yoshino, K. (1993). Experiment on validity of skin resistance level as an index of arousal level. Jpn. J. Ind. Health. 35, 257-268. https://doi.org/10.1539/joh1959.35.257

Yamazaki, M. \& Sugiyama, Y. (2009). Intervention effect of a motivation video for badminton athletes: Examination from watching the motivation video one hour before. Res. J. Sport Perform. 1, 275-288.

Yamazaki, M., Sugiyama, Y., Uchida, W. \& Oda, N. (2008). The intervention effect of the motivation video watching just before the match for badminton players. Jpn. J. Test Meas. Health Phys. Educ., 8, 1725.

Yoshida, K., Sakamoto, Y., Miyaji, I. \& Yamada, K. (2012). Analysis comparison of brain waves at the learning status by simple electroencephalography. IEICE Tech. Rep. 112, 37-42. 


\section{(c) $\underset{\mathrm{EY}}{\mathrm{NO}} \mathrm{\Theta}$}

This work is licensed under a Attribution-NonCommercial-NoDerivatives 4.0 International (CC BY-NC-ND 4.0). 